\title{
Long term effects of cryosurgery on cutaneous sensation
}

\author{
T S SONNEX, R L JONES, A G WEDDELL, R P R DAWBER
}

\begin{abstract}
In a study of the time course and nature of the sensory loss after cryosurgery the forearms of nine normal subjects were treated with liquid nitrogen using freeze times currently employed in clinical practice in Britain. Ability to appreciate touch, pinprick, and cold in the treated areas was tested at regular intervals and biopsy samples taken to investigate the pathogenesis.

Appreciation of all three modalities of sensation was initially reduced in all nine subjects for all freeze times, yet complete recovery occurred in the seven patients completing follow up. This recovery, however, took up to one and a half years for the longest freeze, with even a 10 second freeze taking up to 10 months.

Patients may be reassured that sensory loss after cryosurgery for up to two periods of 30 seconds will almost certainly recover, though it may take up to 18 months to do so.
\end{abstract}

\section{Introduction}

The discovery that cold can produce numbness has been a recurrent process over the centuries. Hippocrates in his Aphorisms ${ }^{1}$ was perhaps the first to record this phenomenon followed by an unknown Anglo-Saxon monk in the eleventh century ${ }^{2}$ and Napoleon's surgeon general in 1807, who noted that amputation could be performed relatively painlessly on soldiers who had unavoidably suffered prolonged contact with Russian snow. Frederick Allen in 1943 restimulated interest with his studies on the use of refrigeration anaesthesia for limb operations. ${ }^{3}$

The analgesic effect of cryosurgery has been proved effectively in the palliative management of various inoperable tumours by direct application to the tumour, ${ }^{4}$ while others have used a cryoprobe to produce analgesia in patients with intractable pain by blocking peripheral nerve function. ${ }^{5}$ These studies have also shown that, although all transmission is blocked in the frozen nerve, full recovery occurs after a variable period. This supports the findings of Lenz, who directly froze the sciatic nerve of nine rabbits with liquid nitrogen ${ }^{6}$ In all cases nerve conduction was completely interrupted, but within 100 days rheobase and chronaxy measurements confirmed full restoration of normal function.

When skin rather than peripheral nerves is frozen it is well recognised that retreatment of the frozen area several days or weeks later will be relatively painless and that pain after cryosurgery is minimal. ${ }^{7}$ Although the studies of Lloyd et al and Barnard et al have provided figures for the duration of pain relief after cryosurgery to various peripheral nerves, ${ }^{5}{ }^{7}$ there is very little information on the duration of sensory loss after cutaneous cryosurgery. Patients, however, may require this

Department of Dermatology, Slade Hospital, Oxford OX3 7JH

T S SONNEX, MB, MRCP, registrar

R L JONES, AIMLS, medical lahoratory scientific officer

A G W'EDDELL, MD, DSC, honorary professor of human anatomy

R P R DAWBER, MA, FRCF, consultant

Correspondence to: Dr T S Sonnex, St John's Hospital for Diseases of the Skin, Leicester Square, London WC2H $7 \mathrm{BJ}$. information, particularly when a sensitive area such as the fingertip is to be rendered anaesthetic by freezing.

Our aim was to determine the changes that occur in cutaneous sensation after the treatment of skin with freeze times currently used in dermatological practice. In particular we wanted to document whether any sensory loss occurring was in fact reversible, and if so how long the different modalities of sensation took fully to recover.

Serial biopsies were performed in an attempt to ascertain the histological changes underlying the clinical findings.

\section{Subjects and methods}

Nine subjects aged 25-78 years participated in the study. Each had four $15 \mathrm{~mm}$ diameter areas of normal skin on the ventral aspect of their right or left forearms treated with 10,20,30, and double 30 second freezes using a CryOwen liquid nitrogen spray gun with a C nozzle held $2 \mathrm{~cm}$ above the skin surface. The double 30 second freeze time had an intervening thaw time of five minutes.

Each freeze was timed from when the treatment field had been defined by the ice margin, the four times being selected as those most frequently used by dermatologists in clinical practice in Britain. The exact site on the forearm for each of the freeze times was varied from subject to subject.

None of the subjects was using any medication-particularly steroids or other anti-inflammatory preparations - that might modify the cryolesion, and no topical agents were applied after freezing.

The ability to appreciate touch (using a wisp of cotton wool), pain (pinprick), and cold (using a cold metal rod) as compared with surrounding, untreated skin was tested on each of the frozen areas at weekly intervals for the first 10 weeks and then at two week intervals until they returned to normal. During these tests the patient's eyes were closed.

Punch bionsy specimens $(2 \mathrm{~mm})$ were taken from some of the treated areas at various intervals after freezing and fixed in $10 \%$ buffered formalin. Wax sections $(5 \mu \mathrm{m})$ were stained with haematoxylin and eosin and Holmes's silver method. Control specimens were taken from surrounding, untreated skin.

\section{Results}

Ability to appreciate all three modalities of sensation tested was initially reduced in all nine subjects for all four freeze times. Complete recovery occurred in all subjects after the 10 and 20 second freezes and in seven subjects after the single and double 30 second freeze times. The remaining two subjects were lost to follow up at 360 and 400 days after freezing.

Ten second freeze (fig 1)-Appreciation of touch returned to normal before that of pain and cold in all cases. This was within 150 days, with a range of 20 to 150 days. Pain appreciation was normal within 150 days in eight subjects (range 40-150 days) and within 300 days in all. Ability to appreciate cold returned to normal within 250 days in all subjects (range 30-250 days). For all three modalities sensation had returned to normal within 70 days in the two youngest subjects (aged 25 and 31 years).

Twenty second freeze (fig 2)-All three modalities of sensation had returned to normal within 300 days in seven subjects and within 380 days in all nine (range 60-380 days).

Single 30 second freeze (fig 3)-Complete recovery occurred in the youngest subject (aged 25) within 150 days. Partial recovery was apparent at 360 and 400 days in the two subjects who were lost to follow up. In the remaining six patients complete recovery occurred within 280 to 500 davs. During the period of recovery appreciation of pain was increased compared with that in normal surrounding skin in two patients. This occurred at 105 and 160 days after freezing resnectively and lasted roughly 40 days. Appreciation of pain was

3

.


subsequently diminished again until recovery had occurred some 120 days later.

Double 30 second freeze (fig 4)-Complete recovery had occurred in seven of the nine subjects within 340 to 550 days. In the remaining two subjects sensation had not completely returned to normal when they were lost to follow up at 360 and 400 days. Two patients experienced increased appreciation of pain at 160 and 200 days after freezing respectively, which lasted for about 50 and 120 days and was then diminished again until complete recovery had occurred some 120 days later. Only one of these patients had a period of increased pain appreciation during recovery from a single 30 second freeze.

Histological findings-Compared with control skin all treated areas sampled within the first few weeks of cryosurgery were found to have an absence of axons in the upper dermis and a noticeable reduction in the deeper dermis. The longer the freeze time the more pronounced were these changes. Even with the longest freeze time, however, Schwann cell and connective tissue pathways were present in normal numbers at all levels, with areas of Schwann cell proliferation. Apart from mild lymphocytic infiltration around a few of the despite normal numbers of Schwann cell pathways. This correlates with the findings of Denny-Brown et al, who showed that on exposing the sciatic nerves of cats to cold functional changes were associated with selective damage to the myelin and axon cylinders. ${ }^{8}$

We also found that after a 10 or 20 second freeze appreciation of touch, pain, and cold returned to normal in all subjects tested within 300 and 380 days respectively. After single and double 30 second freezes complete recovery of the three modalities of sensation occurred in seven of the nine subjects within 500 and 550 days respectively. Substantial though incomplete recovery had also occurred in the remaining two subjects before their default, and we think it quite reasonable to speculate that complete recovery would have occurred within similar time spans.

This full recovery of sensation after complete functional loss correlates with clinical experience and also with the findings after peripheral nerve cryosurgery, ${ }^{9}$ but accurate documentation

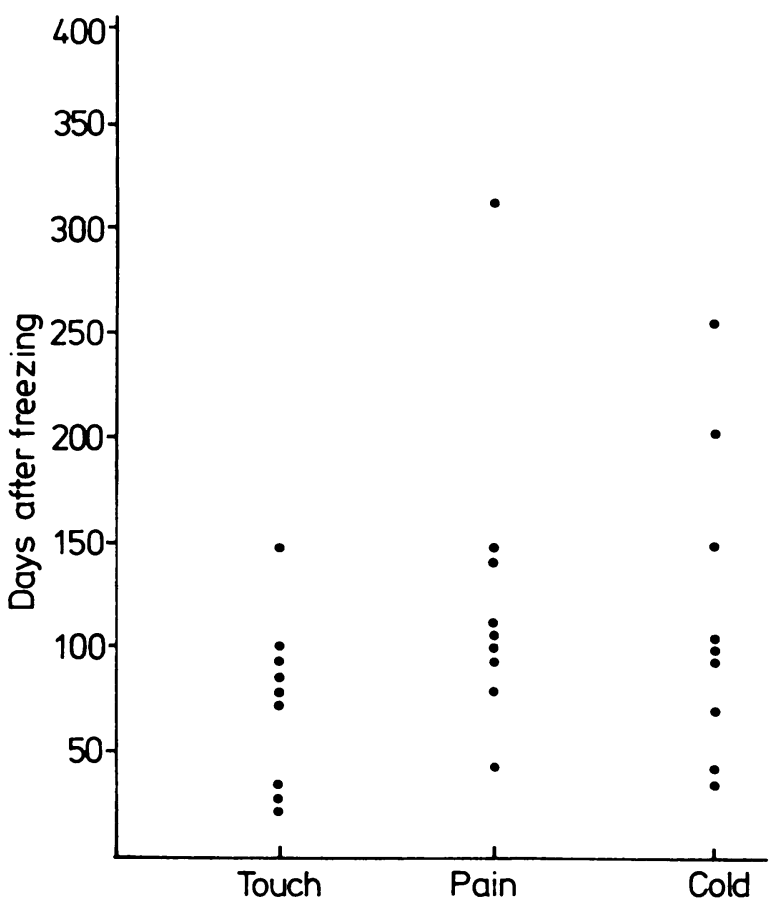

Fig 1

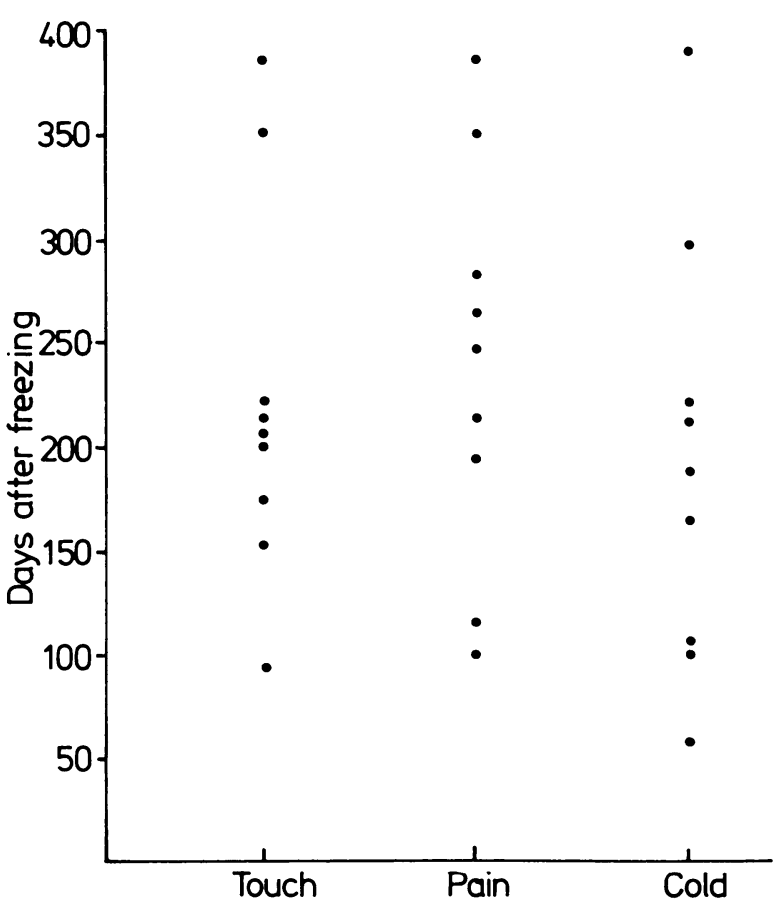

Fig 2

FIG 1 -Recovery of sensation after 10 second freeze. (In this and figs $2-4$ each point represents first day after freezing at which each modality was appreciated as being normal.) FIG 2-Recovery of sensation after 20 second freeze.

neurovascular bundles there was little evidence of inflammation and minimal fibroblastic activity. Dilatation of occasional superficial blood vessels was the only vascular change detected. Biopsy specimens taken at later stages contained increasing numbers of axons at all levels.

\section{Discussion}

Our results indicate that ability to appreciate touch, pain, and cold on ventral forearm skin is reduced by liquid nitrogen spray cryosurgery using freeze times currently employed in Britain. This correlates with clinical findings in that discomfort after cryosurgery is less than with many other surgical techniques and retreatment is often tolerated remarkably well without an anaesthetic. Loss of sensation in areas such as the fingertips, however, may cause considerable concern to the patient.

Histological study of treated areas indicated that the sensory loss was associated with an absence of axons in the upper dermis and noticeably reduced numbers deeper in the dermis, of the time taken for full recovery does not appear to have been reported. Probably it is not widely appreciated that it may take up to one and a half years for complete sensory recovery after a double 30 second freeze and that the sensory deficit caused by even a 10 second freeze may take up to 10 months fully to recover.

Although the necessarily long duration of the study with frequent follow up appointments limited the number of subjects, our general impression was that recovery occurred more rapidly in the younger patients and also that appreciation of touch recovered more quickly than that of pain or cold. This latter finding was commented on by Denny-Brown et al, who found that during freezing touch sensation was also lost before pain sensation. ${ }^{8}$

In view of the underlying histological changes it is perhaps not surprising that sensory function should return completely. Although myelin and axons appear to be quite sensitive to cold, the neural connective tissue sheaths survived apparently unchanged. This has been observed by other workers, who commented that even in the most severe cryosurgically induced pannecrosis the perineurium and epineurium survived. ${ }^{6} 8$ 


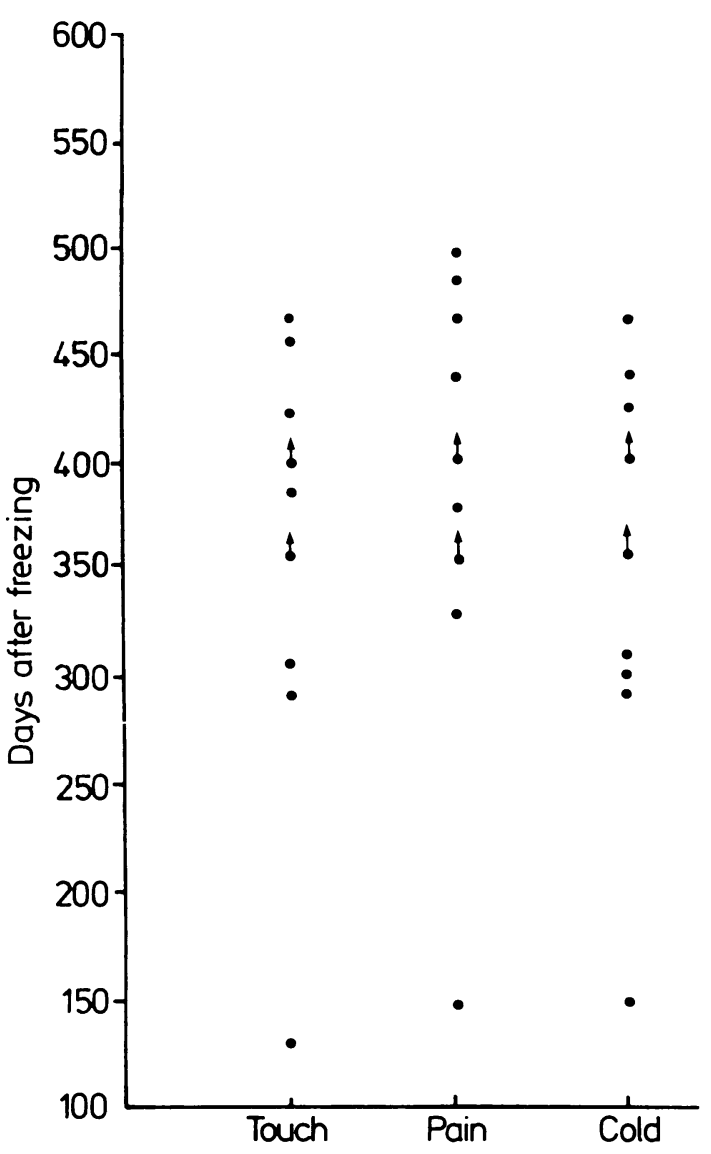

Fig 3

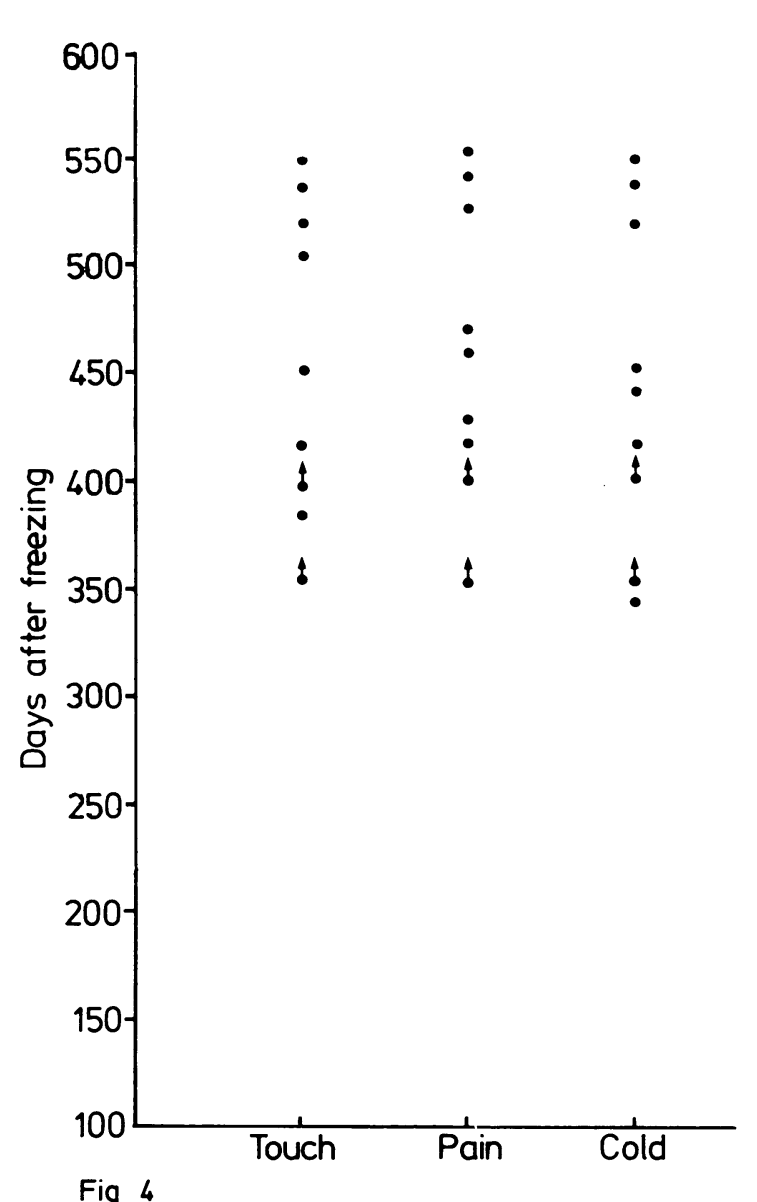

Fig 4

FIG 3-Recovery of sensation after single 30 second freeze. (In this and fig 4 points with arrows represent incomplete recovery at time of default from follow up.) FIG 4-Recovery of sensation after double 30 second freeze.

Shepherd has shown that connective tissue is unaffected by freeze times as used in this study. ${ }^{10}$ This minimal disruption of endoneurium and other connective tissue elements combined with wallerian degeneration with disruption of myelin sheaths and axon disintegration would be classified as a second degree type of nerve injury. ${ }^{11}$ The other important histological feature was the minimal inflammatory reaction, which also has been observed by others. ${ }^{12}$ After a second degree nerve injury an environment where the integrity of the neural connective tissue framework is maintained and inflammation and fibrosis are minimal would be expected to support efficient and complete neural regeneration with consequent full recovery of sensation; this appears to be the case from our clinical and histological findings.

The temporary increase in sensitivity to pain that occurred in three of our patients is more difficult to explain. It may reflect a related transient increase in inflammatory response rather than a scarring process, since none of the patients suffered causalgic pains after full sensory recovery.

In all cases the treated skin had returned to its normal appearance by the time sensation had fully recovered. In particular there was no microscopic evidence of scarring, which further supports the work of Shepherd. ${ }^{10}$

Although further studies may provide more precise figures for recovery time, we think that we can now confidently reassure our patients that sensory loss after cryosurgery for up to two periods of 30 seconds will fully recover-albeit with the proviso that this may take one and a half years.

\section{References} 1 Davison MHA. The evolution of anaesthesia. Br $\mathcal{f}$ Anaesth $1959 ; 31: 134$. Oratton JHG, Singer C. Anglo-Saxo
3 Allen FM. Refrigeration anesthesia for limb operations. Anesthesiology 1943;4:12. Leopard PJ. Cryosurgery and its application to oral surgery. $\mathrm{Br} \mathcal{F}$ Oral Sur $1975 ; 13: 128-52$

. Clow pain relief. Lancet $1976 ;$ ii : $932-4$.

6 Lenz $\mathrm{H}$. Chronaxia and rheobase measurements on the peripheral nerve following cryosurgery. Minerva Med 1974;65:3647.

arnard JDW, Lloyd JW, Glynn CJ. Cryosurgery in the management of intractArf Oral Surg 1978:16:135-42.

Denny-Brown D, Adams RD, Brenner C, Doherty MM. The pathology of injury to nerve induced by cold. F Neuropathol Exp Neurol 1945;4:305.

Beazley RM, Bagley DH, Ketcham AS. The effect of cryosurgery on peripheral nerves. I Surg Res $1974 ; 16: 231-4$.

Shepherd J. Effects of freezing on various dermal connective tissue components. Oxford: University of Oxford, 1979. (MSc thesis.)

11 Sunderland S. Nerves and nerve injuries. Edinburgh and London: Churchill Livingstone, 1968:131.

2 Carter DC, Lee PWR, Johnson RJ. The effect of cryosurgery on peripheral nerve function. Ann $R$ Coll Surg Engl 1972;17:25-31.

(Accepted 2 October 1984)

\section{ONE HUNDRED YEARS AGO}

It seems that only, or chiefly, where there is competition between railway companies working lines to the same place, do the managers provide such necessaries as foot-warmers. This does not speak well for the humanity of railway-directors generally. Only when they think it will increase their popularity and augment their dividends do they bestow any care on the comfort of their passengers. It would be worth while to enact a measure compelling the supply of hot water apparatus of some sort to heat carriages in cold weather. For the majority of those who travel by railway, artificiai heat is as much a necessity as light, and railway-companies ought not to be permitted to economise in the paltry way indicated by the refusal to supply foot-warmers while they enjoy a monopoly. It is most regrettable and humiliating to reflect that, except under the compulsory influence of commercial competition, nothing can be wrung from the managers of our great locomotive systems. We have no patience to comment on the petty parsimony and ungracious indifference displayed by companies that grow rich on a cheeseparing policy which is hurtful to the many and offensive to all. (British Medical fournal 1885;i:189.) 